\title{
AVALIAÇÃO DE SÉRIE DE PACIENTES COM ARTRODESE C1-C2
}

\author{
EVALUATION OF DIFFERENT CASES WITH C1-C2 ARTHRODESIS \\ EVALUACIÓN DE DIFERENTES CASOS CON ARTRODESIS C1-C2
}

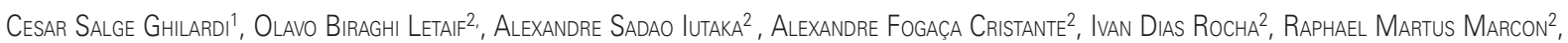
Reginaldo Perilo Oliveira ${ }^{3}$, Tarcísio Eloy Pessoa de Barros Filho ${ }^{4}$

\begin{abstract}
RESUMO
Objetivo: Análise retrospectiva de prontuários de pacientes com instabilidade C1-C2 de causas traumáticas e não-traumáticas, submetidos à artrodese C1-C2. Métodos: Foi realizada análise retrospectiva de prontuários de 20 pacientes do ambulatório de coluna do IOT-HCFMUSP com idades entre 7 e 83 anos (média de 43 anos), de ambos os sexos. Os parâmetros radiográficos para instabilidade foram baseados na medida do intervalo atlanto-axial superior a $3 \mathrm{~mm}$ em adultos e a $5 \mathrm{~mm}$ em crianças, utilizando-se medidas obtidas através de radiografia simples analisada no perfil. Resultados: Foram operados 20 pacientes com instabilidade cervical alta, a maioria de origem traumática. A técnica cirúrgica mais utilizada foi a artrodese descrita por Magerl. Não foram observadas lesões vasculares. Foi registrada complicação infecciosa em dois pacientes. Obteve-se uma taxa de consolidação da artrodese de 85\% e não foram necessárias cirurgias de revisão. Conclusão: Todas as técnicas utilizadas produziram a consolidação óssea satisfatória e foram excelentes para controlar a instabilidade atlanto-axial.
\end{abstract}

Descritores: Coluna Vertebral; Instabilidade articular; Articulação atlanto-axial; Fusão vertebral; Anatomia.

\begin{abstract}
Objective: Retrospective record analysis of patients with C1-C2 instability of traumatic and nontraumatic causes who underwent C1-C2 arthrodesis. Methods: We performed retrospective analysis of medical records of 20 outpatients from the column of IOT-FMUSP aged between 7 and 83 years (mean 43 years) of both sexes. The radiographic parameters for instability were based on measurement of atlanto-axial interval greater than $3 \mathrm{~mm}$ in adults and $5 \mathrm{~mm}$ in children, using measures obtained from lateral plain X-rays. Results: We operated 20 patients with high cervical instability, mostly due to trauma. The surgical technique used was that described by Magerl. There were no vascular injuries. Infectious complications were reported in two patients. We achieved a solid fusion rate of $85 \%$ and no revision surgeries were required. Conclusions: All techniques produced satisfactory bone healing and were excellent for the control of atlanto-axial instability.
\end{abstract}

Keywords: Spine, Joint instability, Atlanto-axial joint, Spinal fusion; Anatomy.

\section{RESUMEN}

Objetivo: Estudio retrospectivo de fichas depacientes con inestabilidad C1-C2, de causas traumáticas y no traumáticas, quienes se sometieron a artrodesis C1-C2. Métodos: Se realizó un análisis retrospectivo de los historiales clínicos de 20 pacientes externos de la columna en el IOT-HC. FM.USP de edades comprendidas entre 07 y 83 años (promedio de 43 años) de ambos sexos. Los parámetros radiológicos de inestabilidad se basaron en la medición del intervalo atlantoaxial superior a $3 \mathrm{~mm}$ en adultos y a $5 \mathrm{~mm}$ en niños, utilizándose medidas obtenidas a partir de radiografías simples analizadas en el perfil. Resultados: Se operaron 20 pacientes con inestabilidad cervical alta, la mayoría con inestabilidad de origen traumático. La técnica quirúrgica más utilizada fue la artrodesis descrita por Magerl. No se observaron lesiones vasculares. Complicaciones infecciosas se registraron en dos pacientes. Hemos logrado una tasa de 85\% de consolidación de la artrodesis y no se requirieron cirugías de revisión. Conclusiones: Todas las técnicas usadas produjeron la consolidación ósea satisfactoria y fueron excelentes para controlar la inestabilidad atlantoaxial.

\section{Descriptores: Columna vertebral; Inestabilidad articular; Articulación atlantoaxial; Fusión vertebral; Anatomía.}

\section{INTRODUÇÃO}

A região cervical superior é composta pelo occipício (occipício ou occipital?, atlas e áxis. Nesta região ocorre metade do movimento de flexão cervical (entre o occipício e C1) e metade do movimento de rotação cervical (C1-C2). A vértebra C1 apresenta uma forma anelar, duas massas laterais, com faces articulares e corpo vertebral ausente.

A vértebra C2 possui corpo vertebral, com processo ósseo dentiforme que se articula com o arco anterior de C1, possui duas massas laterais e duas lâminas.

A estabilidade do segmento atlantoaxial é proporcionada por mecanismos passivos e ativos, que incluem os ligamentos alares, o ligamento cruciforme do atlas, o ligamento apical, o dente do áxis, a cápsula articular, as membranas atlantoaxiais anterior e posterior, a membrana tectórica, a integridade óssea de C1 e C2 e suas facetas articulares. A falha de um ou mais destes mecanismos pode resultar na instabilidade atlantoaxial ${ }^{1}$

Sabe-se que a região cervical superior é acometida frequentemente por doenças que determinam algum tipo de instabilidade, as causas não-traumáticas de instabilidade atlantoaxial compreendem anomalias congênitas do odontoide, neoplasias, processos infecciosos, doenças degenerativas, doenças reumáticas e algumas síndromes ${ }^{2}$

Inúmeras técnicas foram descritas para fixação da região cervical

1. Médico Residente do Grupo de Coluna do Instituto de Ortopedia eTraumatologia do HC/FMUSP - São Paulo, SP, Brasil.

2. Médico Assistente do Grupo de Coluna do do Instituto de Ortopedia eTraumatologia do HC/FMUSP - São Paulo, SP, Brasil.

3. Médico Chefe do Grupo de Coluna do do Instituto de Ortopedia eTraumatologia do HC/FMUSP - São Paulo, SP, Brasil.

4. Professor Titular do do Instituto de Ortopedia eTraumatologia do HC/FMUSP - São Paulo, SP, Brasil.

Trabalho realizado Ambulatório de Ortopedia eTraumatologia da Faculdade de Medicina da USP - São Paulo, Brasil.

Correspondência: Rua Dr. Ovidio Pires de Campos, 333 - Bairro Cerqueira César - São Paulo - Brasil - 05403-010 - cesarsalge@gmail.com 
alta visando à fusão estável entre as vértebras. Em 1910, Mixter e Osgood descreveram a técnica de cerclagem dos processos espinhosos de C1-C2 com seda; em 1939, Gallie descreveu cerclagens nas lâminas de C1-C2. As técnicas de cerclagens apresentam riscos de lesão neurológica inerente à passagem de fios sublaminares, além da necessidade de utilização de órtese externa rígida e de uma alta taxa de não consolidação da artrodese, variável de 9 a $25 \%^{3}$.

Com o desenvolvimento dos sistemas de fixação, surgiram técnicas que permitem fixação mais estável. Os dois métodos mais utilizados hoje são: a técnica de fixação transarticular com parafuso de Jeannert e Magerls ${ }^{4}$ e a técnica de massa lateral de C1 e pedículo de C2 de Harms $^{5}$. Ambas as técnicas, do ponto de vista biomecânico, são superiores à técnica de amarrilho, mas apresentam como riscos: lesões vasculares, hemorragias, sequelas neurológicas e até óbito ${ }^{1,4-7}$.

Wright, em 1998, descreveu uma nova técnica de fixação em $\mathrm{C2}^{6}$. A técnica consiste na passagem de parafusos nas lâminas de C2 e obteve segundo outro trabalho do mesmo autor estabilidade semelhante à das técnicas de Magerl ${ }^{4}$ e Harms ${ }^{5}$ sem as complicações vasculares e neurológicas associadas.

Este trabalho tem como objetivo a avaliação retrospectiva dos pacientes portadores de instabilidade cervical alta de origem traumática e não traumática, submetidos à atrodese C1-C2.

\section{MÉTODOS}

Foram analisados retrospectivamente 20 prontuários de pacientes submetidos à artrodese C1-C2 do ambulatório de coluna do IOT-HCFMUSP.

Foram avaliados pacientes entre 7 e 83 anos de idade, com média de 43 anos, sendo 12 do sexo masculino e 8 feminino e as patologias encontradas foram Artrite Reumatoide, Síndrome de Down, Síndrome de Grisel, fratura-luxação C1-C2, Pseudo-artrose de odontoide distribuídas conforme frequência abaixo demonstrada.

Foram utilizados como critérios de avaliação as seguintes variáveis: presença ou não de déficit; utilização ou não de halo craniano; técnica cirúrgica empregada; complicações; e tempo de consolidação.

Os exames complementares utilizados foram: radiografias nas posições AP, perfil neutro e dinâmico (flexão e extensão máximas) em todos os pacientes (Figura 1). Foi utilizada também a tomografia computadorizada com reconstrução do segmento C1-C2, estes exames também foram realizados nos retornos ambulatoriais para avaliar aos 3 meses se existia alguma perda de material de síntese, aos 6 meses mobilidade anormal na região da artrodese e aos 12 meses consolidação da artrodese.

Os critérios radiográficos de avaliação da instabilidade foram baseados na medida do intervalo atlanto-odontoide, que é o espaço entre a face posterior do arco anterior de $\mathrm{C} 1$ e a face anterior do processo odontoide, considerando-se como instável quando $\geq 5 \mathrm{~mm}$ nos pacientes menores de 15 anos e $\geq 3 \mathrm{~mm}$ nos com mais de 15 anos de idade, vistos nas radiografias em perfil neutro e dinâmico.

A indicação para cirurgia de artrodese C1-C2 foi decorrente da presença de instabilidade cervical alta comprovada radiograficamente ou de alterações neurológicas detectadas ao exame físico clínico.

\section{RESULTADOS}

Dos 20 pacientes operados no nosso serviço a maioria eram do sexo masculino 12 (60\%) e 8 (40\%) eram do sexo feminino. A média de idade foi 43 anos, tendo o doente mais jovem sete anos e o mais velho 83 anos (Tabela 1). Os retornos ambulatoriais ocorreram aos Três, seis e doze meses em todos com radiografias pós-operatórias.

O diagnostico de base foi de etiologia traumática em 12 casos sendo quatro fratura-luxação C1-C2 e oito pseudo-artrose de odontoide, em cinco casos a etiologia foi reumatológica, e em três casos
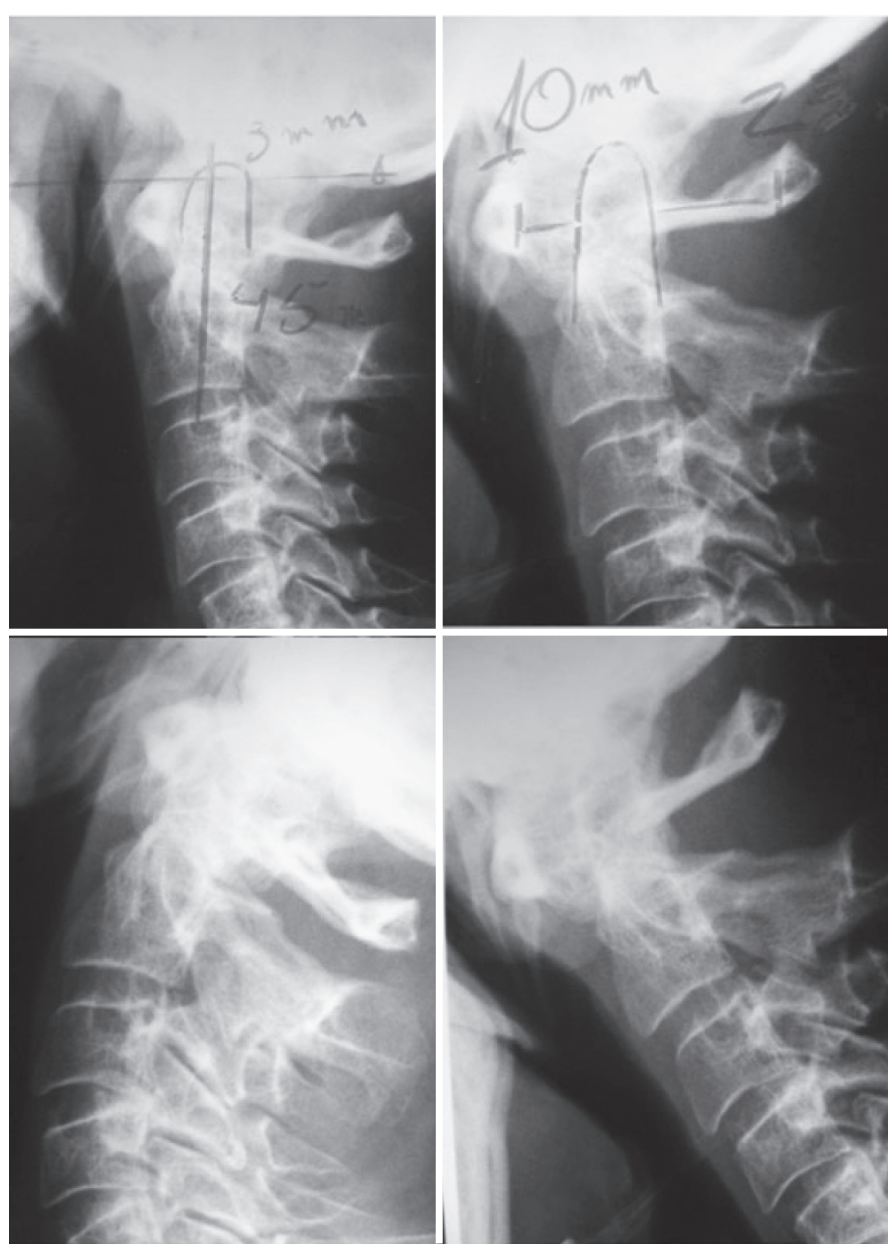

Figura 1. Incidências radiológicas pré-operatórias.

Tabela 1. Frequência de instabilidade cervical de acordo com a faixa etária.

\begin{tabular}{c|c|c}
\hline Idade (anos) & Pacientes & $\%$ \\
\hline $0-20$ & 2 & 10 \\
\hline $21-30$ & 2 & 10 \\
\hline $30-40$ & 5 & 25 \\
\hline $41-50$ & 2 & 10 \\
\hline $51-60$ & 5 & 25 \\
\hline $61-70$ & 1 & 5 \\
\hline $71-80$ & 1 & 5 \\
\hline$>80$ & 2 & 10 \\
\hline
\end{tabular}

foram de etiologias sindrômicas, dois casos de fixação rotatória C1-C2 (Sd. Grisel) e em 01 caso de síndrome de Down (Tabela 2).

A artrodese posterior de Magerl foi o procedimento cirúrgico mais utilizado, seguido do Wright (Tabela 3 ) sendo que em $2 / 3$ dos pacientes submetidos a artrodese de Magerl a etiologia foi pseudo-artrose de odontoide e em 2/3 dos pacientes submetidos ao Wright e etiologia foi artrite reumatoide (Tabela 3)

Aproximadamente 35\% dos pacientes necessitaram da tração halo craniana no pré-operatório (Tabela 4) e 10\% dos pacientes apresentavam déficit, todos de etiologia traumática (Tabela 5).

Não houve registros de complicações vasculares nesta série, não foram registradas lesões neurológicas recentes e ocorreu apenas dois casos de infecção pós-operatória onde não houve a necessidade da retirada do material de síntese.

A avaliação radiográfica pós-operatória revelou boa colocação dos implantes em todos os doentes. Os estudos radiográficos dinâmicos não revelaram mobilidade anormal em nenhum doente, e através da tomografia computadorizada observou-se uma 
Tabela 2. Frequência de instabilidade cervical de acordo com a patologia.

\begin{tabular}{c|c|c}
\hline Patologia & Pacientes & $\%$ \\
\hline Pseudoartrose de odontoide & 08 & 40 \\
\hline Artrite Reumatoide & 05 & 25 \\
\hline Fratura-Luxação C1-C2 & 04 & 20 \\
\hline Síndrome de Grisel & 02 & 10 \\
\hline Síndrome de Down & 01 & 5 \\
\hline
\end{tabular}

Tabela 3. Técnica de artrodese utilizada de acordo com a patologia.

\begin{tabular}{c|c|c|c|c}
\hline Patologia/cirurgia & Magerl & Wright & Galie & Harms \\
\hline Pseudoartrose de odontoide & 6 & 0 & 1 & 1 \\
\hline Artrite Reumatoide & 1 & 4 & 0 & 0 \\
\hline Fratura-Luxação C1-C2 & 1 & 2 & 1 & 0 \\
\hline Síndrome de Grisel & 0 & 0 & 2 & 0 \\
\hline Síndrome de Down & 1 & 0 & 0 & 0 \\
\hline
\end{tabular}

Tabela 4. Frequência de utilização de halo craniano.

\begin{tabular}{c|c|c}
\hline Halo Craniano & Pacientes & $\%$ \\
\hline Com halo & 7 & 35 \\
\hline Sem halo & 13 & 65 \\
\hline
\end{tabular}

Tabela 5. Frequência de pacientes com déficit neurológico.

\begin{tabular}{c|c|c}
\hline Déficit Neurológico & Pacientes & $\%$ \\
\hline Com déficit & 2 & 10 \\
\hline Sem déficit & 18 & 90 \\
\hline
\end{tabular}

taxa de consolidação de $85 \%$ dos casos, em doze meses de acompanhamento. Nos 15\% dos pacientes em que não houve a consolidação, um paciente apresentava pseuartrose de odontoide e realizou a técnica de Magerl e dois apresentavam artrite reumatóide e realizaram a técnica de Wright, em todos os casos não ocorreram repercusões clínicas e/ou neurológicas de instabilidade que indicassem a retirada do material de síntese.

Nenhum paciente necessitou de revisão cirúrgica. Mesmo os casos de complicações infecciosas locais não necessitaram de remoção de implantes.

\section{DISCUSSÃO}

Analisando-se os dados obtidos foi possível observar que em todas as técnicas utilizadas para as diferentes etiologias da instabilidade cervical alta, obteve-se satisfatória taxa de consolidação das artrodeses.

As técnicas modernas de artrodese C1-C2 com uso de parafusos traz benefícios e também riscos para o procedimento. Assim exige-se um profundo conhecimento das técnicas cirúrgicas e um planejamento pré-operatório minucioso.

\section{REFERÊNCIAS}

1. Lau SW, Sun LK, Lai R, Luk MS, Ng YS. Study of the Anatomical Variations of Vertebral Artery in C2 Vertebra With Magnetic Resonance Imaging and Its Application in the C1C2 Transarticular Screw Fixation; Spine 2010, may 15;35 (11): 1136-43.

2. Hensinger, R. N. \& MacEwen, G. D.: "Congenital anomalies of the spine", in Rothman, R.H., Simeone, F.A. et al: The spine, Philadelphia, W.B. Saunders, 1975. Chapter 5, vol.1, p. 157-270.

3. Sorrenti L, lamaguchi MM, Sposeto RB, Araújo MP, lutaka AS, Barros- Filho TEP, et al. Estudo anatômico dimensional do arco posterior de C2 para a instrumentação com parafuso intralaminar. Acta Ortop Bras. 2009;17(4):219-23.

4. Jeanneret B, Magerl F. Primary posterior fusion C1/2 in odontoid fractures: indications, technique, and results of transarticular screw fixation. J Spinal Disord. 1992;5(4):464-75.

5. Harms J. Melcher RP. Posterior C1-C2 fusion with polyaxial screw and rod fixation. Spine. 2001:26(22):2467-71.

6. Wright NM, Lauryssen C. Vertebral artery injury in C1-2 transarticular screw fixation: results of a survey of the AANS/CNS section on disorders of the spine and peripheral nerves. American Association of Neurological Surgeons/Congress of Neurological Surgeons.J Neurosurg. 1998;88(4):634-40.

7. Mandel IM, Kambach BJ, Petersilge CA, Johnstone B, Yoo JU. Morphologic con-
A taxa de consolidação observada no nosso serviço foi semeIhante a relatada em outras séries envolvendo este procedimento ${ }^{8}$. Dickman e Sonntag ${ }^{9}$ relataram uma taxa de consolidação da artrodese de 98\% em 121 pacientes. Haid relatou fusão documentada em $96 \%$ dos 75 pacientes tratados com parafuso transarticular ${ }^{10}$.

Em um estudo da American Association of Neurological Surgeons, foram relatados a colocação de 2.949 parafusos C1-C2 em 1.318 pacientes. A taxa de lesão da artéria vertebral nesse grupo foi de $4,1 \%$ por paciente ou $2,2 \%$ por parafuso colocado. Houve uma taxa adicional de 0,8\% das suspeitas, mas não foi confirmada ${ }^{11}$.

A técnica de Magerl foi a mais utilizada, pois é uma técnica de fixação transarticular de C1-C2 que confere uma estabilização imediata tem vantagem de obter alta taxa de fusão caso seja alcaçada a redução anatômica com bom posicionamento dos parafusos não necessitando de fixação interna adicional nem o uso de colar no pós-operatório ${ }^{12}$, e a técnica de Wright foi a mais utiliza nos pacientes portadores de Artrite Reumatóide devido a destruiçao óssea provocada pela doença causando assim perda de parâmetros anatômicos importantes.

O aumento da taxa de sucesso das artrodeses atlantoaxiais realizadas nos últimos anos pode estar relacionada com o desenvolvimento do diagnóstico por imagem bem como de um desenvolvimento das técnicas cirúrgicas.

Em nossa casuística foi observado dois casos de infecção pós-operatória e não foram observadas lesão da artéria vertebral, falhas do material de síntese ou parafusos mal posicionados.

Neste levantamento duas crianças foram operadas ambas apresentavam Síndrome de Grisel e foi optado pela artrodese de Galie devido dificuldade técnica da passagem de parafusos em C1-C2 em crianças e embora possam ocorrer desvios na coluna cervical sub-axial e restrição de crescimento do canal vertebral nos níveis C1 e C2 após a artrodese atlantoaxial em crianças um mau alinhamento pós-operatório diminui durante o crescimento, possivelmente devido à remodelação da coluna cervical pediátrica, embora a remodelação do diâmetro do canal vertebral não possa ser esperado, realçando a importância da redução anatômica no momento da cirurgia ${ }^{13}$.

Aproximadamente $60 \%$ dos nossos pacientes apresentavam instabilidade cervical alta de origem traumática, não apresentando doenças crônicas ou imunodepressão, o que pode talvez explicar baixas/menores taxas de complicações pós-operatórias.

\section{CONCLUSÕES}

Para diminuir as taxas de complicações cirúrgicas é necessário realizar uma avaliação pré-operatória criteriosa, tornando assim as técnicas modernas de artrodese cervical alta mais segura e com altas taxas de sucesso.

Todas as técnicas utilizadas levaram a uma taxa de consolidação óssea satisfatória e foram excelentes para controlar a instabilidade atlantoaxial e com baixos índices de complicações proporcionando uma alternativa confiável para o tratamento da instabilidade cervical alta.

siderations of $\mathrm{C} 2$ isthmus dimensions for the placement of transarticular screws. Spine. 2000;25(12):1542-7.

8. GlufWM, Schmidt MH, Apfelbaum RI. Atlantoaxial transarticular screw fixation: a review of surgical indications, fusion rate, complications, and lessons learned in 191 adult patients. J Neurosurg Spine; 2005;2(2):155-63.

9. Dickman CA, Sonntag VK. Posterior C1-C2 transarticular screw fixation for atlantoaxial arthrodesis. Neurosurgery. 1998;43(2):275-80.

10. Haid RW Jr. C1-C2 transarticular screw fixation: technical aspects. Neurosurgery. 2001;49(1):71-4

11. Wright NM, Lauryssen C. Vertebral artery injury in C1-2 transarticular screw fixation: results of a survey of the AANS/CNS section on disorders of the spine and peripheral nerves. American Association of Neurological Surgeons/Congress of Neurological Surgeons. J Neurosurg. 1998;88(4):634-40.

12. Wang $C$, Yan M, Zhou H, Wang S, Dang G. Atlantoaxial transarticular screw fixation with morselized autograft and without additional internal fixation: technical description and report of 57 cases. Spine (Phila Pa 1976). 2007:32(6):643-6.

13. Ishikawa M, Matsumoto M, Chiba K, Toyama Y, Kobayashi K. Long-term impact of atlantoaxial arthrodesis on the pediatric cervical spine. J Orthop Sci. 2009;14(3):274-8 